\title{
A Study Of The Antimicrobial Susceptibility Pattern Of Neisseria Gonorrhoeae
}

\author{
Vandana.W.V ${ }^{1}$, N.K.Saxena ${ }^{2}$ \\ ${ }^{I}$ (Assistant professor, Dept of Microbiology, Vydehi institute of Medical Sciences, Bangalore, India) \\ ${ }^{2}$ (Professor, Dept of Microbiology, Gandhi Medical College, Hyderabad, India)
}

\begin{abstract}
Background and objectives: Rapidly emerging antimicrobial resistance of Neisseria gonorrhoeae isolates to currently recommended antibiotics is a setback for effective treatment and control of disease. This study was carried out to determine the antimicrobial susceptibility profile in the isolates obtained from female sexual workers and male patients attending STI clinics in Hyderabad.

Materials and methods: Isolates were tested for sensitivity pattern of penicillin, tetracycline, ciprofloxacin azithromycin, cefixime and spectinomycin. Minimum inhibitory concentration (MIC) and zones of inhibition were interpreted according to CLSI guidelines. $\beta$ lactamase production was detected by chromogenic cephalosporin method using nitrocefin disc.

Results and conclusion: 20 isolates of Neisseria gonorrhea were identified and tested for antimicrobial susceptibility profile. Of them 35\%, 35\%, 20\%, 45\% and 5\% were resistant to penicillin, tetracycline, ciprofloxacin, azithromycin and cefixime respectively. $30 \%$ of isolates were penicillinase-producing $N$. gonorrhoeae. More of less susceptible strains to these antibiotics were identified. Emergence of less susceptible $(10 \%)$ and resistant strains (5\%) to cefixime, which is the current drug of choice, is a cause of concern. Thus periodic surveillance of susceptibility levels of $N$. gonorrhoeae is essential to prevent the dissemination of drug resistant strains in the community.
\end{abstract}

Keywords: Antimicrobial susceptibility, MIC values, Neisseria gonorrhoeae, PPNG

\section{Introduction}

Gonorrhea remains one of the most common sexually transmitted infection (STI) in developing countries. Two important factors, which are responsible for the high rate of prevalence of gonorrhea, are increasing antimicrobial resistance of $\mathrm{N}$. gonorrhoeae and presence of a large reservoir of asymptomatic carriers that unknowingly transmit the disease to their sexual contacts.

Strategies for the control of gonorrhoea have relied on the use of highly effective and often, single-dose therapy administered at the time of diagnosis [1]. Therapeutic options for gonococcal infection are nowadays limited due to the spread of gonococci resistance to a wide variety of antimicrobials. The dramatic increments in the rates of quinolone-resistant gonococci and the current shortage of spectinomycin leave the newer cephalosporins as the last safe choice among the drugs that are currently recommended as firstline treatment. Therefore, increasing reports of reduced in vitro activities of expanded-spectrum cephalosporins against Neisseria gonorrhoeae are of serious concern [2].

Since the failure to cure a case of gonorrhoea has public health implications due to the potential for continued transmission and rapid emergence of antimicrobial resistance and facilitating the spread of HIV, Sentinel surveillance and close monitoring of the in vitro antimicrobial susceptibility of clinical isolates of $\mathrm{N}$. gonorrhoeae has a crucial role in preventing spread of resistant strains and monitoring effective antimicrobial therapy for gonorrhoea thereby providing guidance for appropriate case management $[3,4]$.

\section{Materials And Methods}

Study population: A total of 50 male patients clinically presenting with acute urethritis at STI OP at Gandhi hospital and 125 consecutive female sexual workers (FSW) either asymptomatic or presenting with cervical/vaginal discharge to a sentinel STI clinic in Hyderabad from November 2008 to July 2009 were included in the study.

Study procedure: Informed consent was obtained from all the participants. Three swabs were collected from each patient for Direct Microscopy, culture and Gen -Probe APTIMA GCassay (a second generation Nucleic acid amplification test- NAAT, which utilizes transcription mediated amplification). All samples were processed by standard bacteriological techniques. Bedside inoculation was done on Modified Thayer Martin medium (consisting of GC agar base supplemented with 2\% Horse hemoglobin, Isovital X and antibiotics such as Vancomycin, Colistin, Nalidixic acid, Trimethoprim) and incubated at $35-36.5^{\circ} \mathrm{c}$ in candle extension jar for 2448hrs. Isolates were identified by standard biochemical tests and confirmed by Phadebact Monoclonal Co agglutination and APTIMA GC assay which were performed according to the manufacturer's instructions. 
Colonies were subculture from primary selective medium to a non Selective medium, such as GC-chocolate agar with $1 \%$ supplement, to obtain pure culture for antimicrobial susceptibility testing. Isolates were preserved in trypticase soy broth with $20 \%$ glycerol at $-70^{\circ} \mathrm{c}$.

Antimicrobial Susceptibility Testing: It was performed on GC susceptibility test medium, a standard quality controlled GC agar base medium with 1\% defined supplement (Isovital X). The following commercially available disks (Himedia) were tested by Kirby-Bauer disc diffusion method; Penicillin (10 unit), Tetracycline (30- $\mu \mathrm{g})$, Spectinomycin $(100-\mu \mathrm{g})$, Cefixime $(5-\mu \mathrm{g})$, Ciprofloxacin $(5-\mu \mathrm{g})$, Azithromycin (15- $\mu \mathrm{g})$. MICs of Spectinomycin, Cefixime, Ciprofloxacin, and Azithromycin were determined by the Etest method (AB Biodisk, Sweden). Multiresistant isolates were defined as: quinolone-resistant $\mathrm{N}$. gonorrhoeae (QRNG) + penicillinaseproducing N. gonorrhoeae (PPNG); QRNG+tetracycline-resistant N. gonorrhoeae (TRNG); QRNG + PPNG + TRNG and resistance to any four antibiotics.

The interpretative criteria for all antibiotics except azithromycin were as recommended by the Clinical and Laboratory Standards Institute (CLSI) [5]. The Criteria for interpretation of azithromycin was as recommended by the Neisseria Reference Laboratory (NRL) at CDC [6]. Isolates were tested for $\beta$ - lactamase production by Chromogenic Nitrocefin disk method (BBL Cefinase; Becton Dickinson). ATCC 49226 was used as a positive control strain as recommended by CLSI.

Ethical approval: Study was approved by ethical committee of Gandhi Medical College and Hospital, Hyderabad.

\section{Results}

During the period of eight months, $125 \mathrm{FSW}$ and 50 males attending STI OP were enrolled among whom $13(10.4 \%)$ and $7(14 \%)$ isolates of Neisseria gonorrhea were identified by culture respectively. By NAAT, $18.4 \% \mathrm{FSW}$ and $40 \%$ males had infection. A total of 20 isolates were put up for antimicrobial susceptibility testing. The susceptibility pattern is as shown in Table 1.

All the isolates were sensitive to spectinomycin with $\mathrm{MIC}<4 \mu \mathrm{g} / \mathrm{ml}, 85 \%$ were sensitive to cefixime with MIC $0.023 \mu \mathrm{g} / \mathrm{ml}$. $30 \%$ were $\beta$ lactamase positive (PPNG). Among the $\beta$ lactamase negative isolates, all had reduced susceptibility to penicillin. Among the 6 PPNG strains, two were resistant to ciprofloxacin and azithromycin, one was resistant to tetracycline and cefixime, one was resistant to ciprofloxacin and tetracycline, one was resistant only to cefixime and one was resistant to all the three.

Of the three strains which were less susceptible and resistant to cefixime, one was sensitive to all other drugs, one was resistant to pencillin(PPNG) and ciprofloxacin and one was resistant to pencillin(PPNG), tetracycline and ciprofloxacin. 10\% of strains were resistant to 4 antibiotics, $20 \%$ were resistant to 3 antibiotics, $35 \%$ were resistant to 2 antibiotics and $15 \%$ were resistant to only one antibiotic. $20 \%$ strains were sensitive to all antibiotics except pencillin. Of the multiresistant strains $20 \%, 10 \%$ and $10 \%$ were QRNG+PPNG, QRNG+TRNG and QRNG+PPNG+TRNG.

TABLE -1; Antibiotic susceptibility profile; no and \% (n=20)

\begin{tabular}{|l|l|l|}
\hline Penicillin & $\mathrm{S} ; \geq 47$ & 0 \\
& $\mathrm{LS} ; 27-46$ & $13(65 \%)$ \\
& $\mathrm{R} ; \leq 26$ & $7(35 \%)$ \\
\hline Tetracycline & $\mathrm{S} ; \geq 38$ & $4(20 \%)$ \\
& $\mathrm{LS} ; 31-37$ & $9(45 \%)$ \\
& $\mathrm{R} ; \leq 30$ & $7(35 \%)$ \\
\hline Ciprofloxacin & $\mathrm{S} ; \leq 0.06$ & $7(35 \%)$ \\
& $\mathrm{LS} ; 0.125-0.5$ & $9(45 \%)$ \\
& $\mathrm{R} ; \geq 1$ & $4(20 \%)$ \\
\hline Azithromycin & $\mathrm{S} ; 0.125-0.5$ & $7(35 \%)$ \\
& $\mathrm{LS} ; 0.5-1$ & $4(20 \%)$ \\
& $\mathrm{R} ; \geq 1$ & $9(45 \%)$ \\
\hline Cefixime & $\mathrm{S} ; \leq 0.25$ & $17(85 \%)$ \\
& $\mathrm{LS} ; 0.25-1$ & $2(10 \%)$ \\
& $\mathrm{R} ; \geq 1$ & $1(5 \%)$ \\
\hline Spectinomycin & $\mathrm{S} ; \leq 32$ & $20(100 \%)$ \\
& $\mathrm{LS} ; 64$ & 0 \\
& $\mathrm{R} ; \geq 128$ & 0 \\
\hline
\end{tabular}

(Percentages are according to disk diffusion $(\mathrm{mm})$ for Penicillin and Tetracycline and according to MIC ( $\mu \mathrm{g} / \mathrm{ml})$ values for Ciprofloxacin, Azithromycin, Cefixime and Spectinomycin) 


\section{Discussion}

Resistance of $N$. gonorrhoeae to traditional antimicrobial agents, e.g., penicillin and tetracycline, in the 1980s led to discontinuation of their use in treating gonococcal infections [7]. Our observation also reinforces the emergence of penicillin, tetracycline and quinolone resistance in $N$. gonorrhoeae isolates.

There were more of less susceptible strains than resistant strains for penicillin, tetracycline and ciprofloxacin in the present study similar to a study conducted by Manju bala et al in Delhi [8]. This may reflect the loss of selective pressure from the disuse of these antibiotics as treatment for gonorrhoea. In the present study, PPNG were 30\% which compared well with other studies as shown in Table 2 except for a study from Indonesia which reported 79\%. In WHO Western Pacific Region (WPR), the prevalence of PPNG varied from $1 \%$ to $90 \%$ [8]. PPNG isolates from Asia are proline auxotrophs and carry a 4.4 MDa plasmid [9]. There is an additional conjugative 24.5 MDa large plasmid associated with it. The presence of the associated conjugative plasmid in Asian strain is responsible for rapid spread of resistance to other gonococci. Tetracycline resistance in gonococci may be mediated by chromosomal or plasmid determinants. The location of the tet $\mathrm{M}$ gene on the transferable plasmid has perhaps served to enhance the transmission efficiency of tetracycline resistance in $N$. gonorrhoeae strains, causing the rapid spread of TRNG.

The percentage resistance of $N$. gonorrhoeae isolates to a panel of antibiotics in the present study is compared with various studies in Table 2. Presently, the recommended first-line treatments for gonorrhea in most countries include antibiotics such as cefixime, ceftriaxone, spectinomycin, and in some cases azithromycin or ciprofloxacin. However, resistance to fluoroquinolones and azithromycin is rapidly increasing $[8,10]$. The use of the quinolone group of antibiotics for the treatment of gonorrhoea has been discontinued in India for quite some time because of reported high levels of resistance. However, there were considerable differences in rates of quinolone resistance in different studies in India. Incidence of QRNG (MIC $>1 \mu \mathrm{g} / \mathrm{ml}$ ) was much higher in other studies unlike in the present study (20\%) and in a study by Shilpee et al (10\%) [11] as shown in Table 2. QRNG is also widely distributed in other WHO SEAR and WPR countries [12,13,14, 15, and 16]. Recently and most disquieting, the emergence of $\mathrm{N}$. gonorrhoeae isolates with reduced susceptibility or resistance in vitro to broad-spectrum cephalosporins such as cefixime [7,17, 18, 19, 20,21,22] and ceftriaxone [8] have been reported. In occasional cases, treatment failures when using cefixime have been reported [22]. In the present study also resistant and less susceptible strains to cefixime were noted which is a cause of concern.

It is fortunate that spectinomycin resistance has not been reported often as it is an alternative drug of choice for cases having hypersensitivity to cephalosporins. Spectinomycin is not easily available in India and this may explain the retention of efficacy of this antibiotic. However, some a small number of spectinomycin-resistant strains were found in China, Vietnam [12] and also from India [23]. A study from Indonesia [24] reported $18.1 \%$ resistant strains. Therefore, effective surveillance of antimicrobial resistance is required to monitor trends in established types of resistance and promptly identify new types of resistance.

TABLE 2 - Resistance of antibiotics in various studies

\begin{tabular}{|l|l|l|l|l|l|l|l|}
\hline \multicolumn{2}{|c|}{} & \multicolumn{6}{|c|}{ ANTIBIOTICS WITH THEIR RESISTANCE IN \% } \\
\hline Study & Region & penicillin & tetracycline & ciprofloxacin & cefixime & spectinomycin & PPNG \\
\hline $\begin{array}{l}\text { Manju bala } \\
\text { 2002-2006 }\end{array}$ & Delhi & $54-18$ & $6.7-18.2$ & $80-83$ & $\begin{array}{c}1.7-5.5 \\
\text { (LS) }\end{array}$ & 0 & 21.2 \\
\hline $\begin{array}{l}\text { Sunil sethi } \\
2006\end{array}$ & Delhi & 46.6 & 51 & 77.7 & 0 & $\mathrm{Nt}$ & 22 \\
\hline $\begin{array}{l}\text { DoneganEA } \\
2006\end{array}$ & Indonesia & 81 & 100 & 40 & 0 & 0 & 79 \\
\hline $\begin{array}{l}\text { Yang.Y } \\
2006\end{array}$ & Shanghai & 93 & 56 & 98 & 0 & 0 & 37.8 \\
\hline $\begin{array}{l}\text { Dejongh H } \\
2007\end{array}$ & $\begin{array}{l}\text { South } \\
\text { Africa }\end{array}$ & 32 & 36 & 7 & 0 & $\mathrm{Nt}$ & 16 \\
\hline $\begin{array}{l}\text { Khakhi P } \\
2007\end{array}$ & Delhi & 33.3 & 23 & 98 & 0 & 0 & 20 \\
\hline $\begin{array}{l}\text { Shilpee } \\
2007\end{array}$ & Delhi & 20 & 10 & 10 & nt & 0 & 20 \\
\hline $\begin{array}{l}\text { Present } \\
\text { study }\end{array}$ & Hyderabad & 35 & 35 & 20 & $\begin{array}{l}5 \\
(10-\text { LS })\end{array}$ & 0 & 30 \\
\hline
\end{tabular}

LS; Less susceptible, nt; not tested 


\section{Conclusion}

Our study highlights the need for assessment of antimicrobial susceptibility pattern which is of great importance in monitoring the emergence and spread of resistance and planning of appropriate treatment regimens and control programmes. This also helps in preventing the resistant strains becoming endemic especially when commercial sex workers (high frequency transmitters) have been infected.

\section{Acknowledgements}

We thank all the participants, clinicians and staff who helped in data collection and NARI, Pune, India for Molecular laboratory support. The views expressed herein are those of the authors and does not reflect the views of the funding agency or the other organizations that supported this study.

Conflict of interest: None.

Funding: This study was supported by a grant from Bill and Melinda Gates Foundation.

\section{References}

[1] Rahman M, Alam A, Nessa K, Nahar S, Dutta DK, Yasmin L, et al . Treatment failure with the use of ciprofloxacin for gonorrhea correlates with the prevalence of fluoroquinolone-resistant Neisseria gonorrhoeae strains in Bangladesh. Clin Infect Dis 2001; 32:884-9.

[2] Eva Tzelepi1, Maria Daniilidou, Vivi Miriagou, Eirini Siatravani, Efthimia Pavlidou an Alexandros .Cluster of multidrug-resistant Neisseria gonorrhoeae with reduced susceptibility to the newercephalosporins in Northern Greece.Journal of Antimicrobial Chemotherapy doi:10.1093/jac/dkn236 Advance Access publication 12 June 2008

[3] $\mathrm{P} \mathrm{Khaki}^{1}$, P Bhalla ${ }^{1}$, A Sharma ${ }^{1}$, V Kumar Correlation between In vitro susceptibility and treatment outcome with azithromycin in gonorrhoea: A prospective study Year : 2007 | Volume : 25 | Issue : 4 | Page : 354-357

[4] Manju Bala, Krishna Ray \& S.M. Gupta.Comparison of disc diffusion results with minimum inhibitory concentration (MIC) values for antimicrobial susceptibility testing of Neisseria gonorrhoeae. Indian J Med Res 122, July 2005, pp 48-51

[5] Clinical and Laboratory Standards Institute. Performance standards for antimicrobial susceptibility testing; 15 th informational supplement, M2-A8 and M7-A6. Vol. 25, Clinical and Laboratory Standards Institute 2005. p. 1.

[6] Centers for Disease Control and Prevention. Disk Diffusion Susceptibility Testing: Neisseria gonorrhoeae reference strains for antimicrobial susceptibility testing. Neisseria Reference Laboratory ; Revised 2005

[7] Robert Lindberg, 1 Hans Fredlund, 1 Robert Nicholas, 2 and Magnus Unemo. Neisseria gonorrhoeae Isolates with Reduced Susceptibility to Cefixime and Ceftriaxone: Association with Genetic Polymorphisms in penA, mtrR, porB1b, and ponA $\nabla$. Antimicrob Agents Chemother. 2007 June; 51(6): 2117-2122.

[8] Manju Bala, Krishna Ray, S. M. Gupta, Sumathi Muralidhar and R. K. Jain Changing trends of antimicrobial susceptibility patterns of Neisseria gonorrhoeae in India and the emergence of ceftriaxone less susceptible N. gonorrhoeae strains. Journal of Antimicrobial Chemotherapy (2007) 60, 582-586

[9] Sparling PF. Biology of Neisseria gonorrhoeae. In : Holmes KK, Mardh PA, Sparling PF, Lemon SM, Piot P, et al, Sexually transmitted diseases, 3 rd ed. McGraw Hill: New York; 1999. p. 131-48.

[10] Martin, I. M., S. Hoffmann, and C. A. Ison. 2006. European Surveillance of Sexually Transmitted Infections (ESSTI). The first combined antimicrobial susceptibility data for Neisseria gonorrhoeae in Western Europe. J. Antimicrob. Chemother. 58:587-593.

[11] Shilpee C, Ramachandran VG, Das S, Bhattacharya SN. Antimicrobial susceptibility profile of Neisseria gonorrhoeae at STI clinic. Indian J Med Microbiol 2008;26:62-4

[12] Tapsall JW. Surveillance of antibiotic resistance in Neisseria gonorrhoeae in the WHO Western Pacific Region 2002. Commun Dis Intell 2003; 27: 488-91.

[13] Rahman M, Sultan Z, Monira S et al. Antimicrobial susceptibility of Neisseria gonorrhoeae isolated in Bangladesh (1997 to 1999): rapid shift to fluoroquinolone resistance. J Clin Microbiol 2002 40: 2037-40.

[14] Lesmana M, Lesron CI, Taslim D et al. In vitro antibiotic susceptibility of Neisseria gonorrhoeae in Jakarta, Indonesia. Antimicrob Agents Chemother 2001; 45: 359-62.

[15] Ray K, Bala M, Kumari S et al. Antimicrobial resistance of Neisseria gonorrhoeae in selected World Health Organization Southeast Asia Region countries: an overview. Sex Transm Dis 2005; 32: 178-84.

[16] Trees DL, Sirivongrangson P, Schultz AJ et al. Multiclonal increase in ciprofloxacin-resistant Neisseria gonorrhoeae, Thailand 1998-1999. Sex Transm Dis 2002; 29: 668-73

[17] Australian Gonococcal Surveillance Programme. 2006. Annual report of the Australian Gonococcal Surveillance Programme. Commun. Dis. Intell. 30:205-210. [PubMed

[18] Ito, M., T. Deguchi, K. S. Mizutani, M. Yasuda, S. Yokoi, S. Ito, Y. Takahashi, S. Ishihara, Y. Kawamura, and T. Ezaki. 2005. Emergence and spread of Neisseria gonorrhoeae clinical isolates harboring mosaic-like structure of penicillin-binding protein 2 in Central Japan. Antimicrob. Agents Chemother. 49:137-143

[19] Martin, I. M., S. Hoffmann, and C. A. Ison. 2006. European Surveillance of Sexually Transmitted Infections (ESSTI). The first combined antimicrobial susceptibility data for Neisseria gonorrhoeae in Western Europe. J. Antimicrob. Chemother. 58:587-593

[20] Takahata, S., N. Senju, Y. Osaki, T. Yoshida, and T. Ida. 2006. Amino acid substitutions of mosaic penicillin-binding protein 2 associated with reduced susceptibility to cefixime in clinical isolates of Neisseria gonorrhoeae. Antimicrob. Agents Chemother. 50:3638-3645

[21] Tanaka, M., H. Nakayama, K. Huruya, I. Konomi, S. Irie, A. Kanayama, T. Saika, and I. Kobayashi. 2006. Analysis of mutations within multiple genes associated with resistance in a clinical isolate of Neisseria gonorrhoeae with reduced ceftriaxone susceptibility that shows a multidrug-resistant phenotype. Int. J. Antimicrob. Agents 27:20-26

[22] Wang, S. A., M. V. Lee, N. O'Connor, C. J. Iverson, R. G. Ohye, P. M. Whiticar, J. A. Hale, D. L. Trees, J. S. Knapp, P. V. Effler, and H. S. Weinstock. 2003. Multidrug-resistant Neisseria gonorrhoeae with decreased susceptibility to cefixime-Hawaii, 2001. Clin. Infect. Dis. 37:849-852

[23] Bala M, Ray K, Salhan S. First case of spectinomycin resistant Neisseria gonorrhoeae isolate in New Delhi, India. Sex Transm Infect 2005; 81: 186-7

[24] Joesoef MR, Knapp JS, Idajadi A et al. Antimicrobial susceptibilities of Neisseria gonorrhoeae strains isolated in Surabaya, Indonesia. Antimicrob Agents Chemother 1994; 38: 2530-3. 